\section{No. 4513 April 28, 1956}

In my example of the clock paradox, observer $R$ remains at rest in an inertial frame, that is, he remains in a free path; observer $M$ transfers from one inertial frame to another, that is, he transfers from one free path to another and these different free paths do not combine into a single free path. Once again, this is an absolute distinction between $R$ and $M$.

My purpose was to point out how this absolute distinction results in a distinction between the ways in which $R$ and $M$ describe the relative journey. Therefore, the usual relativistic result cannot, in fact, be made to yield a paradox. My purpose was not to re-establish the usual result. Had it been, I need only have remarked that two curves with the same end-points in general possess different lengths. But it is this obvious result that Dingle now denies.

In spite of what Dingle writes, physicists and mathematicians are quite certain about what follows rationally from the postulates of relativity theory. What no one can be certain about in the same way is the extent to which the actual universe conforms to the postulates. By reason of the many tests of the theory, most of us are convinced that the theory can be correctly applied to the phenomena here considered. However, Dingle might be entitled to question this applicability. What he is not entitled to state is that someone else's calculations "must be wrong" for unspecified reasons.

Berkeley Astronomical Department,

\section{W. M. MCCreA}

University of California.

Prof. McCrea wanders widely from the point, and I do not propose to follow him. Of course, the traveller must be accelerated in order to start, turn and stop again, and this may infinitesimally affect his clock. As McCrea agrees, the accelerations may be extremely brief, so the clock cannot lose much time in them or it would go backwards. The $2 \frac{1}{2}$ years in question is a function of $T$, the time of what he now calls "the free path", during which the engine is cut out. Hence the "absolute" distinction, arising from the use of the engine, is eliminated. McCrea makes no comment on the remarkable feat of $R$ in beating light to a standstill. His remarks abouts geodesics are a wide and needless generalization of the problem; he has said nothing in answer to the definite statements I made.

One or two minor points in his letter require brief comment. First, he says, "The question is not, What has 'happened' to the clocks? It is, What have the clocks measured ?" The question is simply, Will one clock differ from the other or not ? That is all. What they have measured, if anything, is a metaphysical question; it does not enter the problem.

Secondly, McCrea says that I must believe in the classical universal time because I say "you must read them [the clocks] at the same time". My article stated specifically that "the same time" for one observer was not "the same time" for another, so how can this imply belief in the classical universal time?

Thirdly, McCrea calls my paraphrase of Einstein's paper "a travesty" because I have not mentioned "the all-important postulate of the existence of inertial frames of reference". I have looked through Einstein's paper again, and find that he also fails to mention this all-important postulate: he speaks only of "stationary" and "moving" systems, as I did. This may make his paper a travesty of reason, though I do not think so; but I cannot accept it as a criticism of my paraphrase.

Fourthly (and this, of course, is the really serious aspect of the matter), McCrea says, "physicists and mathematicians are quite certain about what follows rationally from the postulates of relativity theory". How, then, does ho explain the number of different solutions of the clock paradox, a few of which he mentioned in his first letter, and how is it that it has been left for him to be the first to discover, after forty-six years, that there is no paradox at all ?

Finally, he says that I am not entitled to state "that someone else's calculations 'must be wrong' for unspecified reasons". I claim that my article did specify the reasons. Herbert DINGLE

\title{
PERFLUOROALKOXY RADICALS
}

\author{
By D. A. BARR, W. C. FRANCIS and DR. R. N. HASZELDINE \\ University Chemical Laboratory, Cambridge
}

$I^{\mathrm{T}}$ $T$ is reasonably well established ${ }^{1}$ that primary alkoxy radicals react either $(a)$ by hydrogen abstraction from a molecule $Q \mathrm{H}$ :

$$
R \mathrm{CH}_{2} \cdot \mathrm{O} \cdot Q \mathrm{H} \longrightarrow R \mathrm{CH}_{2} \mathrm{OH}+Q \cdot
$$

for example,

$$
R \mathrm{CH}_{2} \mathrm{O} \cdot+R \mathrm{CH}_{2} \mathrm{O} \cdot \longrightarrow R \mathrm{CH}_{2} \mathrm{OH}+R \mathrm{CHO}
$$

or $(b)$ by loss of formaldehyde to give a radical of shorter chain-length :

$$
R \mathrm{CH}_{2} \cdot \mathrm{O} \longrightarrow \longrightarrow \mathrm{R} \cdot+\mathrm{HCHO}
$$

It is now clear that perfluoroalkoxy radicals $R \mathrm{CF}_{2} \cdot \mathrm{CF}_{2} \cdot \mathrm{O}$. behave somewhat similarly. Such radicals can abstract hydrogen in a manner analogous to that in $(a)$ above; in absence of compounds containing hydrogen, however, a disproportionation reaction, for example,

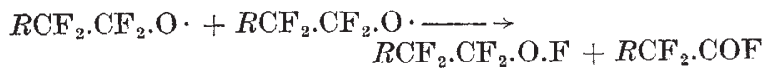

is not observed, and is indeed unlikely, since it would require fission of a $\mathrm{C}-\mathrm{F}$ bond. On the other hand, loss of carbonyl fluoride, the fluorine analogue of formaldehyde, from such radicals :

$$
R \mathrm{CF}_{2} \cdot \mathrm{CF}_{2} \cdot \mathrm{O} \cdot \longrightarrow R \mathrm{CF}_{2} \cdot+\mathrm{COF}_{2}
$$

appears to be a general reaction in fluorocarbon chemistry, and is illustrated by the following three examples.

(1) Photochemical oxidation of a perfluoroiodo. alkane such as $\mathrm{C}_{3} \mathrm{~F}_{7} \mathrm{I}$ is extremely rapid and yields only carbonyl fluoride and (by attack on the glass reaction vessel) silicon tetrafluoride ${ }^{2}$. Facile breakdown of a perfluoroalkyl group by conventional reagents is usually extremely difficult to achieve, and 